\title{
Effectiveness of F-SCORE on the Loser Following Online Portfolio Strategy in the Korean Value Stocks Portfolio
}

\author{
Taegyu Jeong*, Kyuhyong Kim \\ Department of Business Administration, Faculty of Finance, Chung-Ang University, Seoul, Korea \\ Email address: \\ khyongkim@hanmail.net (K. Kim), tgreen1004@naver.com (T. Jeong) \\ ${ }^{*}$ Corresponding author

\section{To cite this article:} \\ Taegyu Jeong, Kyuhyong Kim. Effectiveness of F-SCORE on the Loser Following Online Portfolio Strategy in the Korean Value Stocks \\ Portfolio. American Journal of Theoretical and Applied Business. Vol. 5, No. 1, 2019, pp. 1-13. doi: 10.11648/j.ajtab.20190501.11
}

Received: December 26, 2018; Accepted: January 17, 2019; Published: February 9, 2019

\begin{abstract}
This paper compares the effectiveness of six online portfolio strategies when they are applied to the Korean value stock portfolio. Firstly, using F-SCORE of Piotroski the value stock portfolio is divided into buying group and selling group. Then the six loser following online portfolio strategies are applied for each group and the whole portfolio. RMR strategy for the whole stock portfolio is far superior to the other strategies in terms of the total cumulative return, Sharpe ratio and Calmar ratio. This implies that value stock portfolio has mean reverting or trend following properties that can be utilized by various machine learning techniques.
\end{abstract}

Keywords: Loser Following Online Portfolio Strategies, Machine Learning, F-SCORE, Korean Value Stock Portfolio, Buying Stock Group, Selling Stock Group, Whole Stock Group

\section{Introduction}

Since the study of Fama and French, there has been a trend to invest in stocks with a low market value relative to high book value $[2,3,4]$. However, an empirical study by Piotroski found that only a mere $44 \%$ of stocks with a market-adjusted return over the next two years had a portfolio with high book-to-market stock prices [1]. So, if we could divide these value stocks into strong stocks and weak stocks, could we get better returns? Piotroski draws attention to this point and develops a method of distinguishing between strong and weak value stocks using additional accounting information. When a strategy of buying strong value stocks and selling weak value stocks is conducted, he shows that accounting information is very useful for stock investment.

This study forms a portfolio of value stocks based on the 2007 financial statements in the Korean stock market. Then apply the F-SCORE of Pitotroski to pick up the highest score group of 24 stocks and the lowest score group of 9 stocks. The next step is to apply the online portfolio-loser following strategies to each group and the whole [1]. The 11-year daily price data from April 1, 2007 to September 28, 2018 are analyzed.

This paper shows that the RMR strategy for the whole group is the best in terms of the cumulative rate of return, Sharpe ratio and Calmar ratio. It is not necessary to distinguish between buying and selling groups in the value stock portfolio to get the highest cumulative rate of return once a value portfolio is formed as a basis of online portfolio strategies.

The contribution of this study is that a combination of an accounting information based portfolio selection methodology with machine learning techniques is a valid strategy in the Korean stock market. It is a better idea to confine the portfolio to value stocks when choosing target stocks in implementing online portfolio strategies.

Section 2 explores related works, and Section 3 introduces F-SCORE and six loser following online portfolio strategies. Section 4 shows the results of empirical test. Section 5 is the conclusion.

\section{Related Works}

Fama and French show that the performance of the value portfolio is higher than that of the growth portfolio [2]. They argue that this is because the book to market value ratio (BM) is a variable that indicates the financial distress. Lakonishok, Shleifer, and Vishny also interpret BM as a variable that 
indicates that a company with a high BM is a good performing company, and that its future performance will be good [5]. In reality, analysts do not recommend these high BM stocks as an investment target. Of course, the value of the portfolio is high, but the high performance of individual value stock is not guaranteed.

What is interesting is that there is a lot of information available from financial statements when valuing these value stocks. For example, it is known that we can predict a relatively accurate future stock price pattern from basic changes in the financial statements in the past (leverage, liquidity, profitability, suitability of cash flow, etc.). If we can grasp the intrinsic value of the firm (or the systematic error inherent in market expectations), then the will be able to predict the ultimate loser and winner.

$\mathrm{Ou}$ and Penman and Holthausen and Larcker are examples of studies that try to predict returns using financial statements. They showed that using a variety of financial ratios derived from past financial statements can predict changes in future earnings [7, 8]. However, their limitations are that their methodology is too complicated and that it requires too much historical data. In order to avoid these problems, Lev and Thiagarajan showed that 12 financial signals can be used to predict the current rate of return [9]. Since then, Abarbanell and Bushee have shown that investment strategies using 12 basic signals can achieve significant abnormal returns [10].

On the other hand, recent portfolio selection studies construct online portfolios based on the predicted value of the stocks using machine learning techniques. A study on the composition of the portfolio by machine learning can be seen to originate from the Constant Rebalanced Portfolio and the Universal Portfolio of Cover [11]. After the study of Cover, the Exponential Gradient strategy of Helmbold et al. and the follow the Leader strategy of Gaivoronski and Stella were developed [12-14]. However, it is well known that those follow the leader strategies, which are a kind of momentum strategies, have not performed satisfactorily.

In the 2000s, follow the Loser strategies, contrarian strategies, were developed as an alternative to the follow the Leader strategies. The passive aggressive mean reversion strategy, the Confidence Weighted Mean Reversion strategy, the anti-correlation strategy and Robust Median Reversion strategy are typical contrarian strategies that have proven to be much better than follow the Winner strategies [15-19].

Recently Huang et al. proposed a combination forecasting strategy for online portfolio selection and named it as CFR. They exploit the reversion phenomenon in financial markets by combining several forecasting estimators to improve the prediction accuracy. They claim that CFR overcomes the instability problem of single prediction model. They show that the result of CFR is far better than any single strategy [20].

In the meantime, Pattern Matching Strategy has been developed, various non-parametric strategies led by Gyorfi et al. are quite typical examples. The pattern matching strategy is not to adjust the relative weight by a certain rule but to find a past pattern that is most similar to the current price pattern and construct a portfolio based on the pattern [21-23].

The Meta Learning Algorithm strategy can be seen as a timely strategy that combines the three strategies described above. For example, the Aggregating Algorithm Strategy of Vovk, Online Gradient Updates Strategy of Das and Banerjee, and Online Newton Updates Strategy are categorized as Meta Learning Algorithm strategies [25-28].

Nowadays artificial intelligence (AI) approach is applied to adaptive portfolio management. Obeidat et al. use Long Short Term Memory approach to estimate expected return, volatility and correlation for selected assets and applied Mean-Variance Optimization framework to generate better risk-adjusted returns than conventional passive management [29].

Moreover reinforcement learning is also applied in portfolio management. However, Liang claims that the algorithm demands stationary transition, while the financial market is irregular due to government intervention [24]. They show that the performance of reinforcement learning is still unstable. Generally, it would take a while that artificial intelligence approaches would overwhelm the classical approaches as far as portfolio management is concerned. This study is not aiming at developing a new kind of approach, but rather showing that the performance of online strategy can be improved by adding some accounting information.

\section{Methodology}

\subsection{F-SCORE}

As was mentioned earlier, many studies have shown that companies with high book-to-market ratios (BM firms) have high stock returns. The problem is that only $44 \%$ of high BM companies have positive $(+)$ stock returns in the future. As a result, it is not possible to embody the fact that high BM companies have positive stock price returns in the future as an investment strategy.

To solve this problem, Piotroski developed a screening model that can separate high-return stocks from losers and low-return stocks [1]. He used financial statement data, and identified three characteristics of the company and developed F-SCORE using those characteristics.

The three characteristics of the firm are profitability, financial leverage and liquidity, and operational efficiency. Nine financial variables are selected to represent those characteristics, and each variable is given 1 or 0 depending on whether it had positive impact (1) or negative impact (0) on the price and return of a given firm. The F-SCORE is calculated by adding all the scores of the nine financial variables such that the maximum score is nine, while the minimum score is 0 . Piotroski used F-SCORE to determine the financial status of the target company and made investment decisions. Let's take a closer look at the nine financial variables selected by Piotroski.

\subsubsection{Profitability}

He first selected four variables to measure profitability related factors: ROA, CFO, $\triangle$ ROA, and ACCRUAL. ROA is 
calculated by dividing net profit by the total amount of underlying assets. If the ROA is greater than 0 , then 1 is assigned to the indicator variable F-ROA. If the ROA is less than 0 , then 0 is given to F-ROA. The CFO is calculated by dividing the cash flow from operating activities by the total amount of underlying assets. If the CFO is greater than 0 , then 1 is given to the indicator variable F-CFO. Otherwise, 0 is given to F-CFO. $\triangle$ ROA is the value obtained by subtracting the current ROA from the previous ROA. If $\triangle \mathrm{ROA}$ is greater than 0 , then 1 is assigned to the indicator variable F-ROA, otherwise, 0 is given.

If the net profit is greater than cashflow from operating activities, then it is a negative signal of the future profitability and stock return $[30,31]$. ACCRUAL is adopted to supplement the relationship between net profit and cash flow. ACCRUAL is calculated by dividing (net profit- cash flow from operating activities) by the total amount of underlying assets. If ACCRUAL is less than 0,1 is assigned to the indicator variable F-ACCRUAL, otherwise, 0 is assigned to F-ACCRUAL.

\subsubsection{Leverage and Liquidity}

Three variables were selected to measure the debt repayment ability that is dependent on the capital structure changes: $\triangle$ LEVER, $\triangle$ LIQUID, and EQ_OFFER. As most of the high BM firms experience financial difficulties, we may assume that an increase in leverage, a decline in liquidity, and equity funding to raise funds are bad signals that increase financial risk $[32,33]$. LEVER is calculated by dividing the non-current liabilities by the total amount of the assets. $\triangle$ LEVER is the value obtained by subtracting the previous
LEVER from the current LEVER and the decrease in leverage is interpreted as a positive signal. If $\triangle$ LEVER is less than 0 , then 1 is assigned to the indicator variable F-LEVER, otherwise, 0 is given to F-LEVER. $\triangle$ LIQUID is the value obtained by subtracting the past liquidity ratio from the current liquidity ratio. The increase $\triangle$ LIQUID is interpreted as a positive signal. So that if $\triangle$ LIQUID is greater than 0,1 is assigned to the indicating variable F- $\Delta$ LIQUID. Otherwise 0 is assigned to F- $\triangle$ LIQUID. If new stock is not issued, 1 is assigned to the indicator variable F-EQ_OFFER of EQ_OFFER, If new stock is issued, then 0 is given. This is because the issuance of new stock by the company is interpreted as a negative signal that it lacks the ability to generate internal reserves [32, 33].

\subsubsection{Operational Efficiency}

Finally, we used two variables, $\triangle$ MARGIN and $\triangle$ TURN, to measure the operational efficiency. MARGIN refers to gross profit margin, where the gross margin is divided by sales. $\triangle$ MARGIN is the difference between current MARGIN and previous MARGIN, where positive $\triangle$ MARGIN translates into an increase in gross profit margin. If $\triangle \mathrm{MARGIN}$ is greater than 0 , then 1 is assigned to the indicator variable F-MARGIN, otherwise, 0 is assigned to F-MARGIN. TURN is the total assets turnover, where total sales is divided by total assets. $\triangle$ TURN is obtained by subtracting the prior TURN from the current TURN, and the increase in total asset turnover is interpreted as a positive signal. If $\Delta$ TURN is greater than 0,1 is assigned to the indicator variable F-TURN.

F-SCORE is calculated by summing the nine indicator variables described so far.

$$
\begin{gathered}
\text { F-SCORE }=\text { F-ROA }+ \text { F- } \Delta \text { ROA }+ \text { F-CFO }+ \text { F-ACCRUAL }+ \text { F- } \Delta \text { LEVER }+ \\
\text { F- } \Delta \text { LIQUID }+ \text { F-EQ_OFFER }+ \text { F- } \Delta \text { MARGIN }+ \text { F- } \Delta \text { TURN }
\end{gathered}
$$

F-SCORE is an indicator of how desirable a firm is, and is the sum of the signals of the company's financial status, such that the score ranges between 0 and 9. Piotroski (2000) used this indicator to tell winners from losers.

Piotroski expects that the present F-SCORE is positively related to the future performance and stock price returns, assuming that the current fundamental factors predict future fundamental factors [1]. In particular, by using F-SCORE, he thinks that he can make a better decision than using only one specific variable out of 9 [1].

However, one of the limitations of F-SCORE is that it is possible to lose a lot of information by replacing the values of variables with indicators 0 and 1 . As an alternative, you may want to consider the ordering and summing of the values of each variable. There is also no theoretical background, but simply adding up and using them as investment criteria is a catch-all. However, Piotroski attempted to distinguish between strong and weak companies, so it would be no different from using other complex methods [1].

In this study, Korean stocks (KOSPI) were selected using Piotroski's F-SCORE. We calculated Piotroski's F-SCORE for non-financial listed companies listed in the Kis-Value database. In particular, Piotroski classified the winners and losers every year and used strategies to buy and retain winners for one year and to shorten the losers for one year [1]. However, in this study, we selected value stocks using 2007 financial data and classified the winners and losers by using F-SCORE, then applied the online portfolio strategies 11 years.

\subsection{The Strategies for Benchmark}

Online portfolio should be constructed based on forecasted future prices. Recently, future prices forecasting is done by machine learning. From now on, we will examine various benchmark strategies and follow the loser strategies where forecasting is done by machine learning.

In order to evaluate online portfolio strategies, we need benchmarks. There are three benchmark strategies. The first is the buy and hold strategy, the second is the post-market best stock strategy, and the third is the continuous rebalancing strategy. Let's look at each of them.

\subsubsection{Buy and Hold Strategy}

The buy and hold strategy is the most fundamental 
benchmark strategy for evaluating the performance of a portfolio. The strategy is to initially construct portfolio $b_{1}$ and keep it until the last moment of cashing. Therefore, the value $S_{n}$ of the portfolio at the time of cashing can be expressed as follows. $x_{t}$ is the rate of return at time $\mathrm{t}$.

$$
S_{n}\left(B A H\left(b_{1}\right)\right)=b_{1} \cdot\left(\bigodot_{t=1}^{n} x_{t}\right)
$$

For example, if you invest $1 / \mathrm{m}$ each in $\mathrm{m}$ kinds of shares, the portfolio will be

$$
\mathrm{b}_{1}=\left(\frac{1}{\mathrm{~m}}, \cdots, \frac{1}{\mathrm{~m}}\right)
$$

And $b_{1}$ does not change until it is cashed. This is called a uniform BAH strategy, which is a strategy that follows the market index.

\subsubsection{Best Stock Strategy}

This strategy is an ex post de facto strategy that is not practically possible and is used only as a benchmark to evaluate the portfolio's performance. The strategy selects the best stock from the cashed out portfolio ex post, such that $100 \%$ is invested in that stock.

$$
b^{o}=\underset{b \in \Delta m}{\arg \max } b \cdot\left(\bigodot_{t=1}^{n} x_{t}\right)
$$

The final value of the best stock portfolio is calculated as follows.

$$
S_{n}(\text { Best })=\max _{b \in \Delta m} b \cdot\left(\bigodot_{t=1}^{n} x_{t}\right)=S_{n}\left(B A H\left(b^{o}\right)\right)
$$

\subsubsection{Constant Rebalanced Portfolio}

The third benchmark strategy is constant rebalanced portfolio (CRP) strategy where the weight for each stock at every rebalancing is kept constant. Such that we must rebalance the portfolio in order to keep the investment weight constant.

$$
b_{1}^{n}=\{b, b, \cdots\}
$$

For example, if we maintain a constant investment weight for each stock at each portfolio adjustment, $b$ is defined as the Uniform Constant Rebalanced Portfolio (UCRP).

$$
b_{1}=\left(\frac{1}{m}, \cdots, \frac{1}{m}\right)
$$

For UCRP, the value of the accumulated portfolio after $n$ periods is as follows.

$$
S_{n}(C R P(b))=\prod_{t=1}^{n} b^{T} x_{i}
$$

We can get ex post $b^{*}$ that maximizes $S_{n}(C R P(b))$ using the following convex function.

$$
b^{*}=\underset{b^{n_{\in}} \in m}{\operatorname{argmax}} \log S_{n}(C R P(b))=\underset{b \in \Delta m}{\operatorname{argmax}} \sum_{t=1}^{n} \log \left(b^{\prime} x_{t}\right)
$$

The CRP strategy with $\mathrm{b} *$ is called the Best Constant Rebalanced Portfolio (BCRP). This portfolio is practically impossible because we can get the best portfolio only ex post. The final cumulative portfolio value of the BCRP and the corresponding exponential growth rate are defined as follows:

$$
\begin{gathered}
S_{n}(B C R P)=\underset{b \in \Delta m}{\operatorname{argmax}} S_{n}(C R P(b))=S_{n}\left(C R P\left(b^{*}\right)\right) \\
W_{n}(B C R P)=\frac{1}{n} \log S_{n}(B C R P)=\frac{1}{n} \log S_{n}\left(C R P\left(b^{*}\right)\right)
\end{gathered}
$$

Cover proposed to use BCRP returns as benchmark returns because BCRP returns are better than any of the following returns. The return of the best stock strategies, the geometric mean return of the value line index, the average return of Dow Jones Index and the return of the buy and hold strategy [11]. In addition, Cover suggested to use BCRP as a benchmark because BCRP has the same rate of return even when the order of prices is changed [11].

\subsection{The Loser-Following Strategy}

The BCRP strategy assumes i.i.d returns. However, the i.i.d assumption does not always hold in real world. If the i.i.d. assumption does not hold, follow the winner strategy cannot have good performance consistently. Stock prices in real life tend to show a pattern that good performance in one period is followed by bad performance in the next period [34-36]. That is, the stock price shows a pattern that returns to the average rather than the i.i.d.

If the return to the average is assumed, stocks with good performance in this period will have bad performance in the next period, and stocks with bad performances in this period will have good performance in the next period. Therefore, it would be a better strategy to reduce the weight of investment in good stocks and to increase the weight of investments in bad stocks in this period. The strategy that reduces the weight of investment in the winners and increase the weight of investment in losers of this period is called follow the loser strategy. There are many kinds of follow the loser strategies. In this paper, we look at Anti Correlation Strategy, Passive Aggressive Mean Reversion Strategy, Confidence Weighted Mean Reversion Strategy, Online Moving Average Strategy, and Robust Median Reversion Strategy.

\subsubsection{Anti-Correlation Strategy}

The anticor strategy of Borodin et al. is the first to appear as a follow the loser strategy. Assuming that market prices follow an average regression, they proposed an anticor strategy, where the cross-correlations have a positive value and the autocorrelation has a negative value [37, 38]. Firstly, the log value of the relative price matrix of the first window and the second window is defined by the following equation.

$$
y_{1}=\log \left(x_{t-2 w+1}^{t-w}\right)
$$

and

$$
y_{2}=\log \left(x_{t-w+1}^{t}\right)
$$

Here, $x_{t-w-1}^{t}$ is the relative price matrix $(\mathrm{w} \times \mathrm{m})$ from time $\mathrm{t}-\mathrm{w}-1$ to time $\mathrm{t}, \mathrm{m}$ is the number of stocks constituting the portfolio, $\mathrm{w}$ is the window size. Once the matrices $y_{1}$ and $y_{2}$ are given, the cross-correlation coefficient matrix between $y_{1}$ and $y_{2}$ is obtained by the following equation. 


$$
\begin{gathered}
M_{c o v}(i, j)=\frac{1}{w-1}\left(y_{1, \mathrm{i}}-\bar{y}_{1}\right)^{\prime}\left(y_{2, \mathrm{i}}-\bar{y}_{2}\right) \\
M_{c o r r}(i, j)=\left\{\begin{array}{cc}
\frac{M_{c o v}(i, j)}{\sigma_{1}(i) * \sigma_{2}(j)} & \sigma_{1}(i), \sigma_{2}(j) \neq 0 \\
0 & \text { otherwise }
\end{array}\right.
\end{gathered}
$$

Where stock $\mathrm{i}$ belongs to the first window and stock $\mathrm{j}$ belongs to the second window. Once the cross-correlation coefficient matrix is obtained, the next step is to adjust the investment proportion according to the average regression-based trading strategy.

Let's denote claim $_{i \rightarrow j}$ as the transfer of the investment from the stock $\mathrm{i}$ in the first window to the stock $\mathrm{j}$ in the second window. The transfer is done when $\bar{y}_{1}(i)>\bar{y}_{2}(j)$ and $M_{\text {corr }}(i, j)>0$ hold, and $\operatorname{claim}_{i \rightarrow j}=M_{\text {corr }}(i, j)+A(i)+$ $A(j)$, where $\mathrm{A}(\mathrm{h})=\left|M_{\text {corr }}(h, h)\right|$ when $M_{\text {corr }}(h, h)<$ 0 , otherwise 0 . When claim $_{i \rightarrow j}$ is obtained, $b_{t+1}(i)$ is calculated using the following equation. $b_{t+1}(i)$ is the component vector of the next period portfolio.

$$
b_{t+1}(i)=b_{t}(i)+\sum_{j \neq i}\left[\text { transfer }_{j \rightarrow i}-\text { transfer }_{i \rightarrow j}\right]
$$

Here

$$
\text { transfer }_{i \rightarrow j}=b_{t}(i) \cdot \frac{\operatorname{claim}_{i \rightarrow j}}{\sum_{j} \operatorname{claim}_{i \rightarrow j}}
$$

The most important variable in Anticor is $w$, the size of the window. We may be able to get the window size that gives the highest return ex post, but we can not get it a priori. We have to choose the window size arbitrarily.

Of course, you may be able to learn the size from a lot of experience, but if the fluctuation is so great, there is no guarantee that you can choose the best size even when we have a lot of experience.

Also Anticor has to use arbitrary correlation as we do have to choose arbitrary length of period to calculate the correlations. It is for sure that we can not take full advantage of the characteristic of average regression. However, at the time when Anticor was announced in 2003, it had the best empirical results.

\subsubsection{Passive Aggressive Mean Reversion (PAMR)}

The basic idea of PAMR is that if the previous rate of return is greater than the threshold, then we expect the rate of return in the next period will be negative, so that the proportion of the investment is reduced in proportion to the rate of increase in the previous period [15]. If the rate of return is smaller than the threshold, then we expect the rate of return will be zero in the next period, so that we keep the share of investment as it is as before. To explain this in more detail, let's first explain the loss function.

$$
\ell_{\varepsilon}\left(b ; x_{t}\right)=\left\{\begin{array}{cc}
0 & b \cdot x_{t} \leq \varepsilon \\
b \cdot x_{t}-\varepsilon & \text { otherwise }
\end{array}\right.
$$

Where $0 \leq \varepsilon \leq 1$ is a sensitivity parameter that is given externally and controls the threshold of average regression. If the loss function is ' 0 ', the portfolio is maintained as it is, and if the loss function is positive, then the portfolio is readjusted so that the loss function becomes ' 0 '. In other words, PAMR gets the portfolio of the next period solving the following optimization problem.

$$
b_{t+1}=\underset{b \in \Delta m^{2}}{\operatorname{argmax}_{1}}\left\|b-b_{t}\right\|^{2} \text { s.t } \ell_{\varepsilon}\left(b ; \boldsymbol{x}_{t}\right)=0
$$

Solving this optimization problem, we reconstruct a new portfolio following equation (19). (Li et al. 2012, Proposition 1).

$$
b_{t+1}=\underset{b \in \Delta m^{2}}{\operatorname{argmax}_{1}}\left\|b-b_{t}\right\|^{2} \text { s.t } \ell_{\varepsilon}\left(b ; \boldsymbol{x}_{t}\right)=0
$$

According to this equation, the proportion of investment is decreased for stocks with a higher return than the average return, and increased for stocks with lower return than the average return. In some cases, the investment proportion may have a negative value. To take this into consideration, the simplex projection step is sometimes adopted [39].

Like Anticor, PAMR has rather a very weak theoretical background. However, in 2012 when PAMR was announced, it performed better than any other algorithms. One drawback of PAMR is that in the absence of an average regression in the next period, performance can be very bad in terms of risk management. Borodin et al. and Li et al. showed very poor performance when this method was applied to DJIA $[15,18$, $37,38]$.

\subsubsection{Confidence Weighted Mean Reversion (CWMR)}

$\mathrm{Li}$ et al. proposed a confidence-weighted mean-regression strategy (CWMR) that estimates the weight of a new portfolio using not only the average weight but also the variance of the existing portfolio [16]. The basic idea is that the portfolio vector $\mathrm{b}$ itself has a multivariate normal distribution, that is, a variance-covariance matrix $\sum \in \mathbb{R}_{m * m}$ with a non-diagonal term of zero and average vector $\mu \in \mathbb{R}_{m}$.

Of course, whenever the new information arrives, the mean and variance are modified to obtain $b_{t} \in \mathrm{N}\left(\mu_{t}, \sum_{t}\right)$, and $b_{t+1}$ is obtained using all available information at time t. In other words, we apply next period's portfolio weight to the current period and check if probability that the rate of return $\left(\mu \cdot x_{t}\right)$ is smaller than $\epsilon$ is greater than threshold $(\theta)$. If the probability is greater than $\theta$ we do not change the portfolio, otherwise, we have to change the portfolio. Therefore, the optimization problem is defined as follows.

$$
\begin{gathered}
\left(\mu_{t+1}, \sum_{t+1}\right)=\underset{\mu \in \Delta m, \Sigma}{\operatorname{argmin}} D_{K L}\left(\mathcal{N}(\mu, \Sigma) \| \mathcal{N}\left(\mu_{t}, \sum_{t}\right)\right) \\
\text { s.t. } \operatorname{Pr}\left[\mu \cdot \boldsymbol{x}_{t} \leq \varepsilon\right] \geq \theta
\end{gathered}
$$

To solve this optimization problem, Li et al. (2013) transformed using two methods. The first transformed optimization problem is as follows [19]. 


$$
\begin{gathered}
\left(\mu_{t+1}, \sum_{t+1}\right)=\operatorname{argmin} \frac{1}{2}\left(\log \left(\frac{\operatorname{det} \sum_{t}}{\operatorname{det} \sum}\right)+\operatorname{Tr}\left(\sum_{t}^{-1} \sum\right)+\left(\mu_{t}-\mu\right)^{\tau} \sum_{t}^{-1}\left(\mu_{t}-\mu\right)\right) \\
\text { s.t. } \varepsilon-\mu^{\top} \boldsymbol{x}_{\boldsymbol{t}} \geq \phi \boldsymbol{x}_{t}^{\top} \sum \boldsymbol{x}_{t}, \mu^{\top} 1=1, \mu \geq 0
\end{gathered}
$$

Solving this optimization problem gives us the following solutions. (Li et al.'s proposition 4.1) [19].

$$
\mu_{t+1}=\mu_{t}-\lambda_{t+1} \sum_{t}\left(x_{t}-\bar{x}_{t} 1\right), \sum_{t+1}^{-1}=\sum_{t}^{-1}+2 \lambda_{t+1}+1 \phi x_{t} x_{t}^{\top}
$$

In this equation, $\lambda_{t+1}$ is the Lagrangian multiplier [19].

$$
\overline{x_{t}}=\frac{1^{\top} \Sigma_{t} x_{t}}{1^{\top} \Sigma_{t}}
$$

Represents the confidence weighted price relative average.

From equation (22), we can see that we use the mean reversion trading strategy, and use the first and second moments of the portfolio vector.

Since $\Sigma$ is a positive semi-definite matrix, it can be decomposed. In other words, given the eigenvalues $\left(\lambda_{1}, \cdots, \lambda_{m}\right)$ of $\Sigma$ and

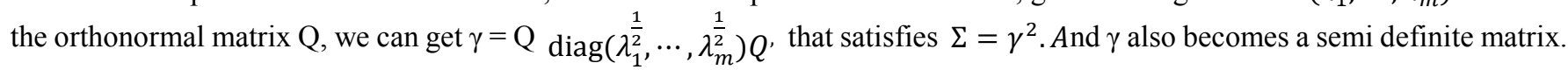
In this case, the optimization problem is defined as follows.

$$
\begin{gathered}
\left(\mu_{t+1}, \gamma_{t+1}\right)=\underset{\mu, \gamma}{\operatorname{argmin}} \frac{1}{2}\left(\log \left(\frac{\operatorname{det} \gamma_{t}^{2}}{\operatorname{det} \gamma^{2}}\right)+\left(\operatorname{Tr}_{t}^{-2} \gamma^{2}\right)\right)+\frac{1}{2}\left(\left(\mu_{t}-\mu\right)^{\prime \gamma_{t}^{-2}}\left(\mu_{t}-\mu\right)\right) \text { s.t. } \varepsilon-\log \left(\mu-x_{t}\right) \geq \phi\left\|\gamma \cdot x_{t}\right\|, \gamma \text { is PSD } \\
\mu \cdot 1=1, \mu \geqslant 0
\end{gathered}
$$

Solving this optimization problem gives us the following solutions. (Li et al.'s Proposition 4.2)[19].

$$
\mu_{t+1}=\mu_{t}-\lambda_{t+1} \sum_{t} \frac{x_{t}-\overline{x_{t}} \cdot 1}{\mu_{t} \cdot x_{t}}, \sum_{t+1}^{-1}=\sum_{t}^{-1}+\lambda_{t+1} \phi \frac{x_{t} x_{t}^{\prime}}{\sqrt{U_{t}}}
$$

Where

$$
\sqrt{U_{t}}=\frac{-\lambda_{t+1} v_{t} \phi+\sqrt{\lambda_{t+1}^{2} v_{t}^{2} \phi^{2}+4 v_{t}}}{2}
$$

Here $V_{i}=x_{t}^{\prime} \sum_{t} x_{t}$ and $\sqrt{U_{t}}=\frac{-\lambda_{t+1} v_{t} \phi+\sqrt{\lambda_{t+1}^{2} v_{t}^{2} \phi^{2}+4 v_{t}}}{2}$ represents the variance of $t$ and $t+1$ trading day respectively. $\lambda_{t+1}$ is the Lagrangian multiplier and the confidence weighted average $\overline{x_{t}}$ is defined as $\bar{x}_{t}=\frac{1^{\prime} \Sigma_{t} x_{t}}{1^{\prime} \Sigma_{t} 1}$

Like Anticor and PMAR, CWMR also uses mean reversion, which makes it difficult to explain theoretically. When CWMR was announced, it was treated to be better than PAMR, which uses only average. However, since CWMR also uses a single period mean reversion, there is no guarantee that the results will necessarily be better when data do not exhibit the characteristics of single period mean reversion.

\subsubsection{Online Moving Average Reversion}

As we have seen, PAMR and CWMR assume a single-period mean reversion, so that applying this method to actual data does not produce such a successful result. Li et Hoi proposed an online portfolio strategy using multi-period mean reversion called OLMAR (Online Moving Average Reversion) [18].

If we look closely at PAMR and CWMR, we implicitly assume $\hat{p}_{t+1}=p_{t-1}$. In other words, the assumption is that daily prices vary extensively. Mostly actual data do not conform to this extreme assumption. To overcome these shortcomings, Li et al. proposed a multi-period mean reversion that uses mean reversion and multi-period moving averages [18].

First, given the price $p_{i}$ vector and window size w, we use the following simplest moving average to predict the relative return of the next period.

$$
M A_{t}=\frac{1}{w} \sum_{i=t-w+1}^{t} P_{i}
$$

Then the relative price of the next period by Li and Hoi's Eq. (1) is as follows [18].

$$
\hat{x}_{t+1}(w)=\frac{M A_{t}(w)}{P_{t}}=\frac{1}{w}\left(1+\frac{1}{x_{t}}+\cdots+\frac{1}{\bigodot_{i=0}^{w-2} x_{t-i}}\right)
$$

Here, $\odot$ denotes the product of the elements. It is possible to increase the size of the window to reflect all the historical prices, but according to the empirical analysis, as the window size increases, the performance decreases [18].

The moving average when considering the whole data, not the window, is obtained by the following equation.

$$
\operatorname{EMA}(\alpha)=\alpha P_{t}+(1-\alpha) E M A_{t-1}(\alpha)=\alpha P_{t}+(1-\alpha) \alpha P_{t-1}+(1-\alpha)^{2} \alpha P_{t-2}+\cdots+(1-\alpha)^{t-1} P_{1}
$$

Where $\alpha \in(0,1)$ is decaying factor. Using $\operatorname{EMA}(\alpha)$, the next period's relative price is obtained by the following equation. 


$$
\hat{x}_{t+1}(\alpha)=\frac{E M A_{t}(\alpha)}{P_{t}}=\frac{\alpha P_{t}+(1-\alpha) E M A_{t-1}(\alpha)}{P_{t}}=\alpha 1+(1-\alpha) \frac{E M A_{t-1}(\alpha)}{P_{t-1}} \frac{P_{t-1}}{P_{t}}=\alpha 1+(1-\alpha) \frac{\hat{x}_{t}}{x_{t}}
$$

Whichever method is used, once $\hat{x}_{t+1}(w)$ is obtained, we can use either PAMR or CWMR. Li and Hoi applied PAMR and called it online moving average reversion (OLMAR) [18]. That is, as in PAMR, we obtain bt +1 using the following equation.

$$
b_{t+1}=\underset{b \in \Delta m}{\operatorname{argmin}} \frac{1}{2}\left\|b-b_{t}\right\|^{2} \text { s.t } b \cdot \hat{x}_{t+1} \geq \varepsilon
$$

It differs from PAMR in that it uses the moving average and obtain the composition of the portfolio based on it. OLMAR is also known to have achieved the best results that were available in 2012 [18]. OLMAR got best performance where PAMR and CWMR failed [18].

\subsubsection{Robust Median Reversion}

All of the strategies discussed so far are likely to distort the estimation results because they take both noise and outliers in the data into consideration. That is, there is a possibility that the performance of the portfolio is distorted because the noise and outliers in the data. An attempt to exclude the effects of these noises and outliers was made by Huang, which is called Robust Median Reversion (RMR) strategy.

The basic idea of the RMR is to find themedian estimate $L_{1}$ at time $\mathrm{t}$ using the following equation: $\hat{P}_{t+1}=L_{1 \text { med } t+1}(w)=\mu_{t+1}$. Where $\mathrm{w}$ is the window size and $\mu_{t+1}$ is obtained by the following equation (Weber 1929, Fermat-Weber problem).

$$
\mu_{t+1}=\operatorname{argmin}_{\mu} \sum_{i=0}^{w-1}\left\|p_{t-1}-\mu\right\|
$$

Where $\|\cdot\|$ denotes Euclidean distance. That is, $L_{1 \text { median }}$ is the value when the sum of Euclidean distances is minimized, where $\mathrm{k}$ price vectors are given. In this case, if the data are not linearly dependent, there is a unique solution. Therefore, the expected relative price using $L_{1 \text { median }}$ is given as follows.

$$
\hat{x}_{t+1}(w)=\frac{L_{1 m e d_{t+1}} m(w)}{P_{t}}=\frac{\mu_{t+1}}{P_{t}}
$$

Once an estimate of the price at time $t+1$ is given, the RMR constructs the portfolio in the same manner as we did in PAMR or OLMAR [18]. In other words, we optimize the following equation.

$$
b_{t+1}=\underset{b \in \Delta d}{\operatorname{argmin}} \frac{1}{2}\left\|b-b_{t}\right\|^{2} \text { s.t } \mathrm{b} \cdot \widehat{x_{t}} \geq \varepsilon
$$

Empirically, RMR has shown better performance than any other algorithms for almost all data [19].

\subsection{Performance Evaluation}

The first criteria for assessing the performance of online portfolio is the final cumulative return. Since the original investment size is normalized as $1\left(S_{0}=1\right), S_{n}$ is the final accumulation size. Of course, the bigger $S_{n}$ is, the better the strategy is.

Another performance measure is (APY $\left.=\sqrt[y]{S_{n}}-1\right)$ where $\mathrm{y}$ is the number of years corresponding to the $\mathrm{n}$ trading days. Of course, the larger the $S_{n}$, the larger the APY, so that they can be regarded as the same standard.

Since we rebalance online portfolios on daily basis, it is essential to evaluate the risk and return-to-risk ratio of Sharpe $[40,41]$. The volatility risk $(\sigma)$ is annualized, and the risk adjusted return like Sharpe ratio is also annualized $[40,41]$.

That is, the Sharpe ratio is obtained by $\operatorname{SR}\left(=\frac{A P Y-R_{f}}{\sigma}\right.$ when the risk-free interest rate $R_{f}$ is given. Of course, the higher the Sharpe ratio, the better the performance of the trading strategy [40, 41].

The drawdown measures how much the current cumulative rate of return has fallen from the past maximum cumulative rate of return [42].

Given, the cumulative return series at each point $\left(S_{1}, S_{2}, \cdots S_{n}\right)$, the reduction rate at time $\mathrm{t}$ is defined by $\mathrm{DD}(\mathrm{t})=\max \left[0, \max _{i \in(0, n)} S_{(i)}-S_{t}\right]$. The maximum drawdown (MMD) is maximum from among the $\mathrm{DD}(\mathrm{t})$ and is defined as $\operatorname{MMD}(\mathrm{n})=\max _{i \in(0, n)}[D D(t)]$. This is a $\operatorname{good}$ methodology for measuring the downside risk of online portfolio strategy. The smaller the MDD, the lower the downside risk.

Calmar Ratio is defined as $\mathrm{CR}=\frac{A P Y}{M D D}$ and shows the annual return over the maximum reduction rate. That is, the larger the APY and the smaller the MDD, the better the performance.

In order to test the performance of the strategy, we divide the return of the portfolio into the return related to the benchmark and the return not related to the benchmark [43]. To do this, we obtain a simple regression equation with the daily excess return of the strategy as the dependent variable and the excess return of the benchmark as the independent variable. $\left(s_{t}-s_{t}(F)\right)=\alpha+\beta\left(s_{t}(B)-s_{t}(F)\right)+\epsilon$, where $s_{t}$ is the rate of return of the strategy, $s_{t}(F)$ is the risk free rate of return and $s_{t}(B)$ is the rate of return of the market index. If $\alpha$ has a statistically significant positive value, it can be judged that the reliability of the online portfolio strategy is very high. If $\beta$ is greater than 1 and statistically significant, then the higher return of the portfolio is not from a simple luck.

\section{Empirical Analysis}

Following Piotroski's methodology, we classified all non-financial companies listed in the Kis-Value database in 2007 into 10 groups. The criterion for the classification was book-to-market value ratios (BM). We selected 33 high $\mathrm{BM}$ stocks at the top $10 \%$.

For those 33 high BM stocks (i.e. value stocks), we applied Piotroski's F-Score to divide them into buying ( 8 to 9 ) and selling ( 0 to 1$)$ groups. As is shown in Table 1 the buying group is composed of 24 stocks, and the selling group is composed of 9 stocks. 
Table 1. Constructing of Portfolios with F-Score.

\begin{tabular}{lll}
\hline rtfolio Name & F-Score & Number of shares \\
\hline Buying Group & 8.9 & 24 \\
Selling Group & 0.1 & 9 \\
Whole Group & $0.1,8.9$ & 33 \\
\hline
\end{tabular}

We applied various loser following online portfolio strategies to the buying group, selling group and the whole group. The data is composed of 2,851 trading days' adjusted closing prices from April 1, 2007 to September 28, 2018. Table 2 shows the cumulative returns over the ten years of four basic benchmarks' strategies (market, uniform, best stock, BCRP) and following the loser strategies (PAMR, CWMR, OLMAR and RMR).

Table 2. The cumulative return of the loser following online portfolio strategies by groups.

\begin{tabular}{llll}
\hline Strategy & Whole & Buying & Selling \\
\hline Market & 2.9803 & 3.1644 & 2.5418 \\
Uniform & 4.3051 & 4.1802 & 4.2815 \\
Best Stock & 9.0544 & 9.0544 & 6.7148 \\
BCRP & 16.4546 & 14.7797 & 11.2722 \\
PAMR & 40.7166 & 56.3599 & 13.6239 \\
CWMR-V & 65.2073 & 88.8332 & 17.1204 \\
CWMR-S & 65.2205 & 89.2317 & 17.1345 \\
OLMAR-S & 1196.00 & 1191.50 & 115.8473 \\
OLMAR-E & 745.406 & 2109.90 & 77.0120 \\
RMR & 2514.80 & 1418.50 & 126.434 \\
\hline
\end{tabular}

The cumulative returns of the two most profitable strategies in bold.

Table 2 shows that following the loser strategies are superior to the basic strategies for every group. From the perspective of long-term investment, we can see that we have to choose following the loser strategies rather than basic strategies. Among following the loser strategies,
RMR and OLMAR are the most profitable strategies, while CWMR and PAMR are inferior strategies. This implies that the next day returns to the mean assumption employed by CWMR and PAMR does not reflect the reality, while the five day return to the mean of OLMAR and RMR does reflect the reality.

In Korea, as in other countries, the performance of the RMR strategy is superior to that of any other strategies [19]. For the RMR strategy, it is much better to apply it to the whole than to apply it to buying and selling groups separately. In other word, the long-term outcome of the RMR strategy is better when it does not distinguish between groups using accounting information. If we have to divide them into two groups, we can see that the performance of buying group is better than that of selling group. This is because the regression to the mean of the buying stock group is more evident than the average regression of the selling stock group.

Figure 1 shows the cumulative gross return pattern for the whole group. RMR has the best cumulative return performance. And OLMAR-S and OLMAR-E are the next best performers respectively. The cumulative return of RMR began to exceed the cumulative return of OLMAR-S and OLMAR_E at around 850 trading days, and this trend continues until the last trading day. Once a strategy started to overwhelm, other strategies could not reverse the trend afterward.

On the other hand the volatility of RMR is much larger than that of OLMAR, but the upward trend is so large that the sharp ratio is not likely to differ greatly from each other. In practice, it will be a big challenge whether RMR can tolerate such volatility despite the upward trend of cumulative returns.

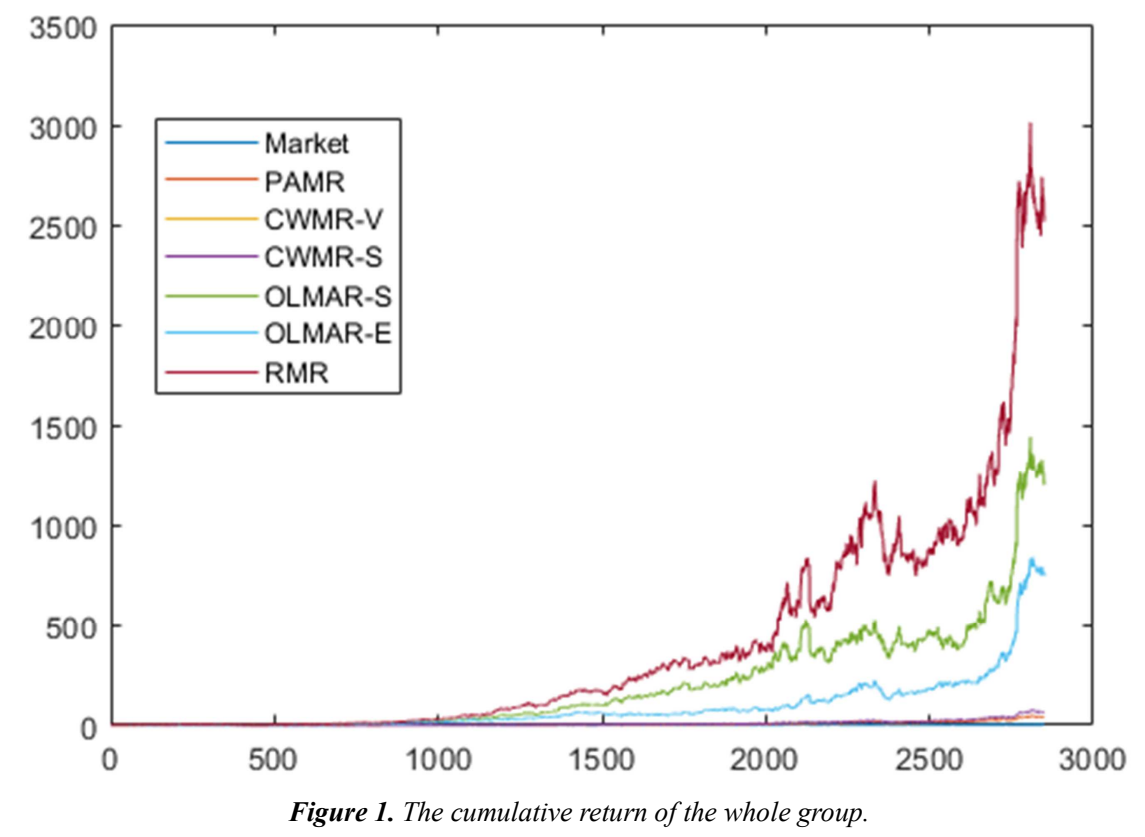

For the buying group in Figure 2, OLMAR-E shows the best cumulative return, while RMR and OLMAR-S are the next best performers. The most remarkable point is that OLMAR-S has the best cumulative return performance until 2100 days, and RMR and OLMAR-S have very similar cumulative return performance. However, OLMAR-E maintains best cumulative return performance 
with a very large difference until the last trading day. That is, OLMAR-S should be selected at first but OLMAR-E should be selected afterward to have the best performance. If you do not want to change the strategy in the middle, you'd rather select RMR strategy, which has consistently good cumulative performance over the whole trading period.

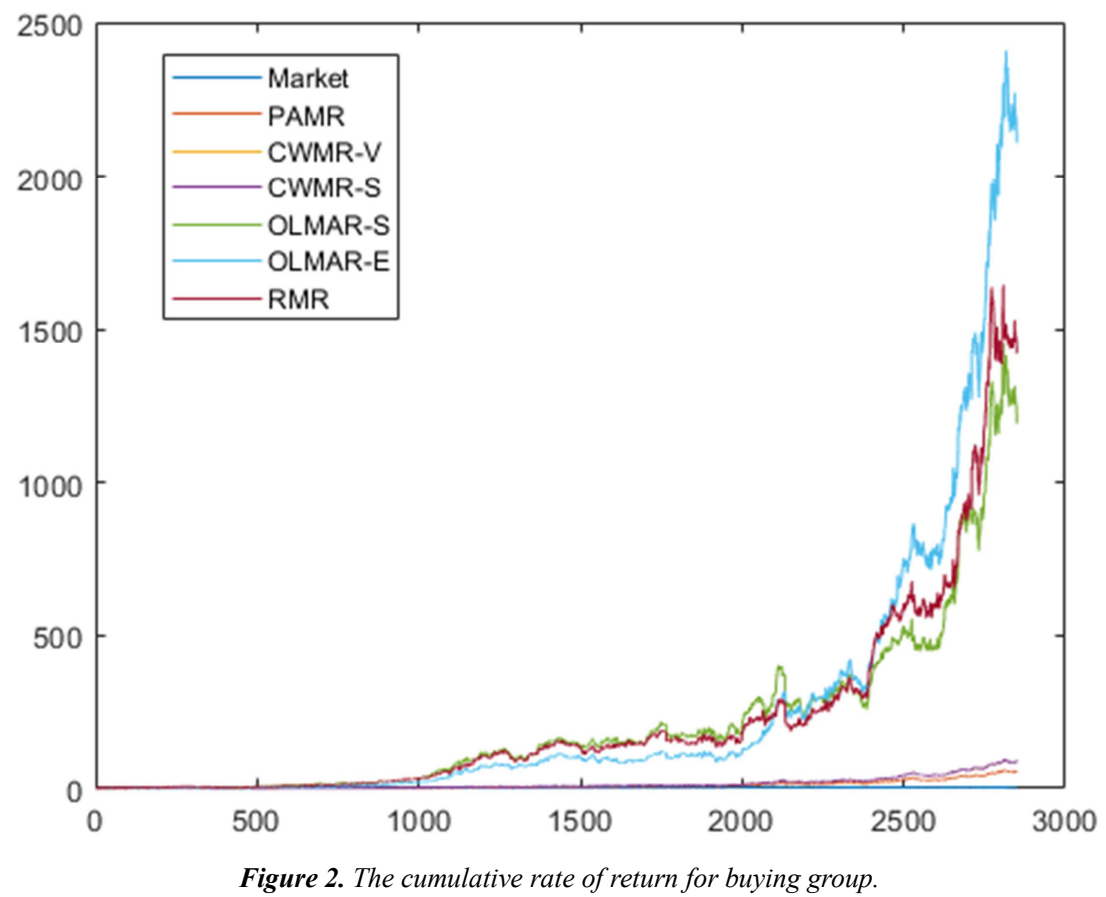

For the selling group in the figure 3, the RMR has the best final cumulative return, while OLMAR-S and OLMAR-E have the next best cumulative returns. However their 11 years of trading performance pattern are very similar to each other. In the case of selling group, the stock prices are expected to have a steady downward pattern resulting in downward moving average. This may force follow the loser strategies to have exaggerated fluctuation.

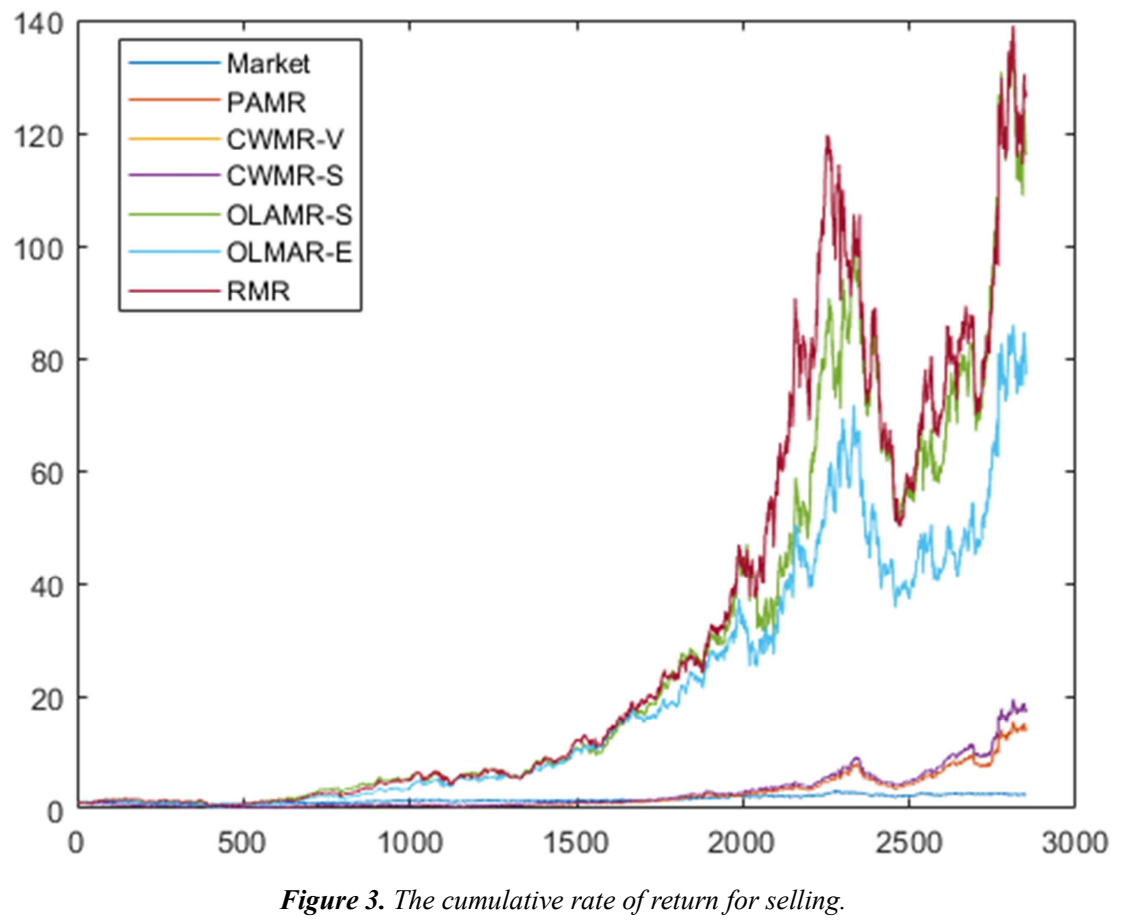

Table 3 shows the winning rates of all strategies are between $50 \%$ and $53 \%$. All $\beta$ coefficients are greater than 1 and statistically significant, which indicate that the rate of return of the strategies above the market rate is not simply due to luck. This interpretation is reinforced by the negative signs of all $\alpha$ coefficients. 
Table 3. The regression on the performance of each strategy.

\begin{tabular}{|c|c|c|c|}
\hline Features & Buying Group & Selling Group & Whole Group \\
\hline Total trading days & 2851 & 2851 & 2851 \\
\hline The market average return (Benchmark) & 0.00111 & 0.000892 & 0.001045 \\
\hline PAMR average return & 0.019768 & 0.004779 & 0.014282 \\
\hline PAMR winning rate & 0.531042 & 0.508593 & 0.53946 \\
\hline$\beta 1$ & 9.499396 & 4.037767 & 7.272506 \\
\hline$\alpha 1$ & -14.3057 & -4.29594 & -10.4799 \\
\hline T-Statistics1 & 49.35281 & 78.31946 & 49.14247 \\
\hline$\rho$-Value1 & 0.00 & 0.00 & 0.00 \\
\hline CWMR-V average return & 0.031159 & 0.006005 & 0.022872 \\
\hline CWMR-V winning rate & 0.537355 & 0.509295 & 0.543669 \\
\hline$\beta 2$ & 14.10343 & 4.938937 & 10.90435 \\
\hline$\alpha 2$ & -21.7435 & -5.2506 & -16.0479 \\
\hline T-Statistics2 & 45.663 & 73.16106 & 45.84819 \\
\hline$\rho$-Value2 & 0.00 & 0.00 & 0.00 \\
\hline CWMR-S average return & 0.031298 & 0.00601 & 0.022876 \\
\hline CWMR-S winning rate & 0.537355 & 0.509295 & 0.543669 \\
\hline$\beta 3$ & 14.16349 & 4.938718 & 10.89656 \\
\hline$\alpha 3$ & -21.8393 & -5.25042 & -16.0343 \\
\hline T-Statistics3 & 45.64476 & 73.0806 & 45.81434 \\
\hline$\rho$-Value3 & 0.00 & 0.00 & 0.00 \\
\hline OLMAR-S average return & 0.417924 & 0.040634 & 0.419502 \\
\hline OLMAR-S winning rate & 0.525079 & 0.507892 & 0.535251 \\
\hline$\beta 4$ & 196.9162 & 49.50736 & 242.1685 \\
\hline$\alpha 4$ & -299.186 & -53.7872 & -359.704 \\
\hline T-Statistics4 & 45.59244 & 126.3328 & 59.81326 \\
\hline$\rho$-Value4 & 0.00 & 0.00 & 0.00 \\
\hline OLMAR-E average returm & 0.740056 & 0.027012 & 0.261454 \\
\hline OLMAR-E winning rate & 0.531743 & 0.50228 & 0.537005 \\
\hline$\beta 5$ & 258.1571 & 33.94083 & 101.7783 \\
\hline$\alpha 5$ & -415.855 & -35.6815 & -146.589 \\
\hline T-Statistics5 & 32.42573 & 150.2631 & 39.58982 \\
\hline$\rho$-Value5 & 0.00 & 0.00 & 0.00 \\
\hline RMR average returm & 0.497545 & 0.044347 & 0.882076 \\
\hline RMR winning rate & 0.529639 & 0.512101 & 0.537706 \\
\hline$\beta 6$ & 208.6969 & 55.76158 & 458.3175 \\
\hline$\alpha 6$ & -319.4 & -61.0716 & -732.053 \\
\hline T-Statistics6 & 38.97511 & 136.136 & 53.67906 \\
\hline$\rho$-Value6 & 0.00 & 0.00 & 0.00 \\
\hline
\end{tabular}

Independent variable is market index rate of return, the six dependent variables are the rate of return of each of the six strategies. $t$-statistic for beta is not reported. However every beta show the value that is greater than 1, which implies that results are not just out of luck.

Figure 4 shows that the six loser following strategies have relatively higher volatility than the rest four benchmark strategies. This suggests that the higher return pattern that is possible by active portfolio strategies is accompanied by higher volatilities. The volatility of the six loser following strategies are at a similar level with no significant difference may imply that we may have to choose the strategy that shows highest cumulative return performance.

MDDs for the 10 strategies are shown in Figure 5. We see that the MDD of the buying group is lower than that of the other groups. The figure clearly shows that we have to suffer from high Maximum draw down possibilities if we want to have higher cumulative rate of return. In addition, selling group has higher drawdown possibilities than other groups. And it would be a better idea to limit our portfolio to buying group only if we do not want to suffer from maximum draw down.

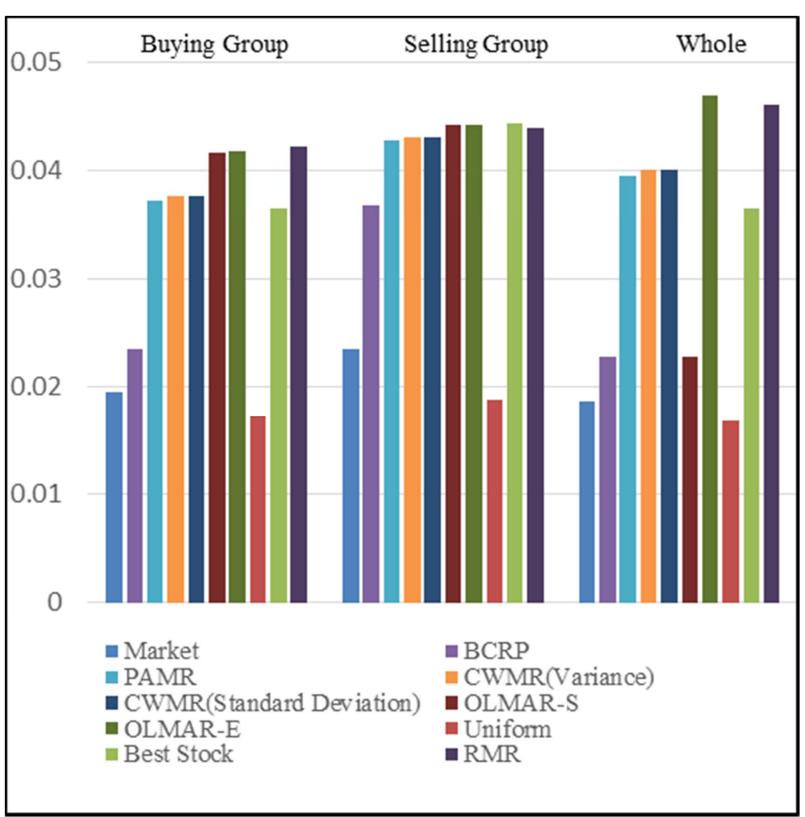

Figure 4. Volatility. 


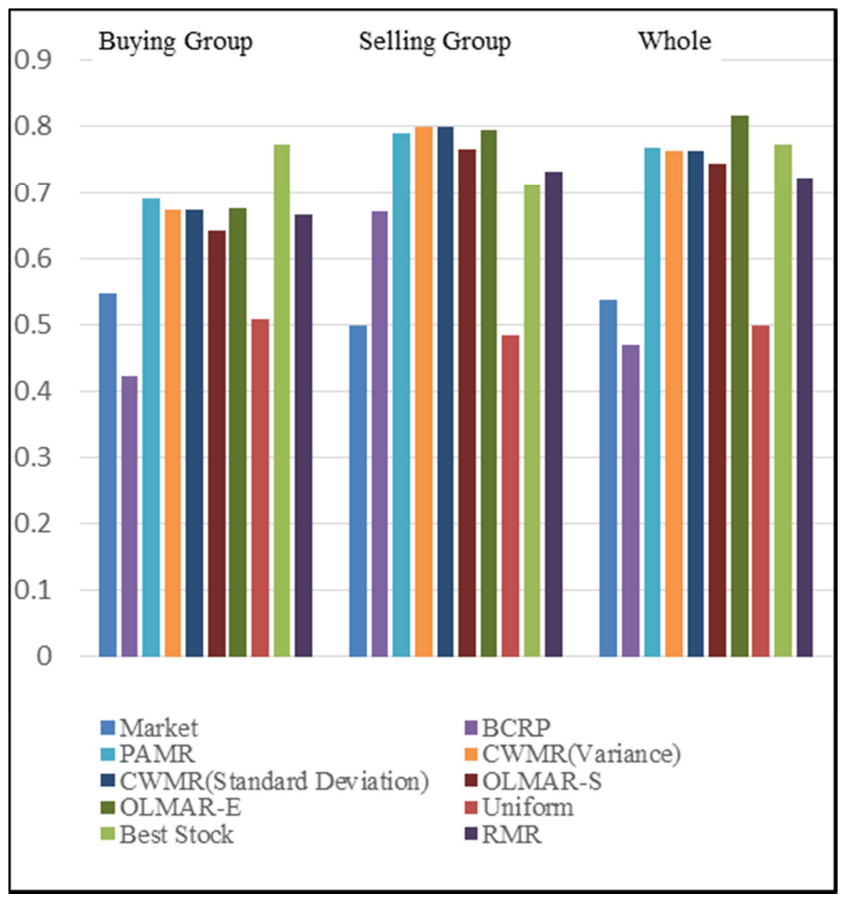

Figure 5. $M D D$.

Figure 6 shows the Sharpe ratios for the 10 strategies, where return and risk are considered simultaneously. OLMAR-E has the highest Sharpe ratio in the buying group, RMR has the next. The RMR shows the highest Sharp ratio in the selling as well as the whole group, OLMAR-S has the next. CWMRs show poor Sharpe ratios. We have seen that RMR and OLMAR have very good cumulative rate of return patterns in Figure 1, and that they have high volatility. Sharpe ratio shows that risk taking through OLMAR and RMR are very good strategies when the return is considered together with risk.

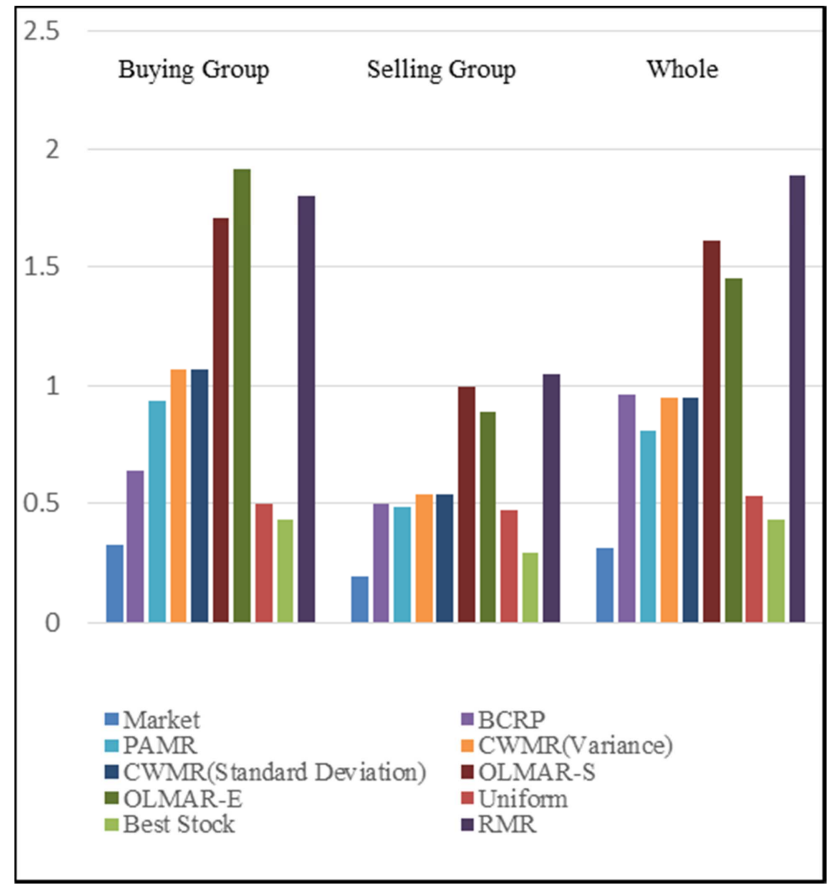

Figure 6. Sharpe Ratio.
Figure 7 shows the Calmar ratio, which is very similar to the Sharpe ratio results in Figure 4. OLMAR-E shows the highest rate of Calmar in the buying group, OLMAR-S shows the next. The RMR shows the highest Calmar in the selling group as well as the whole group, OMMAR-S is the next. Empirically, RMR and OLMAR show good Calmar ratio regardless of the portfolio groups.

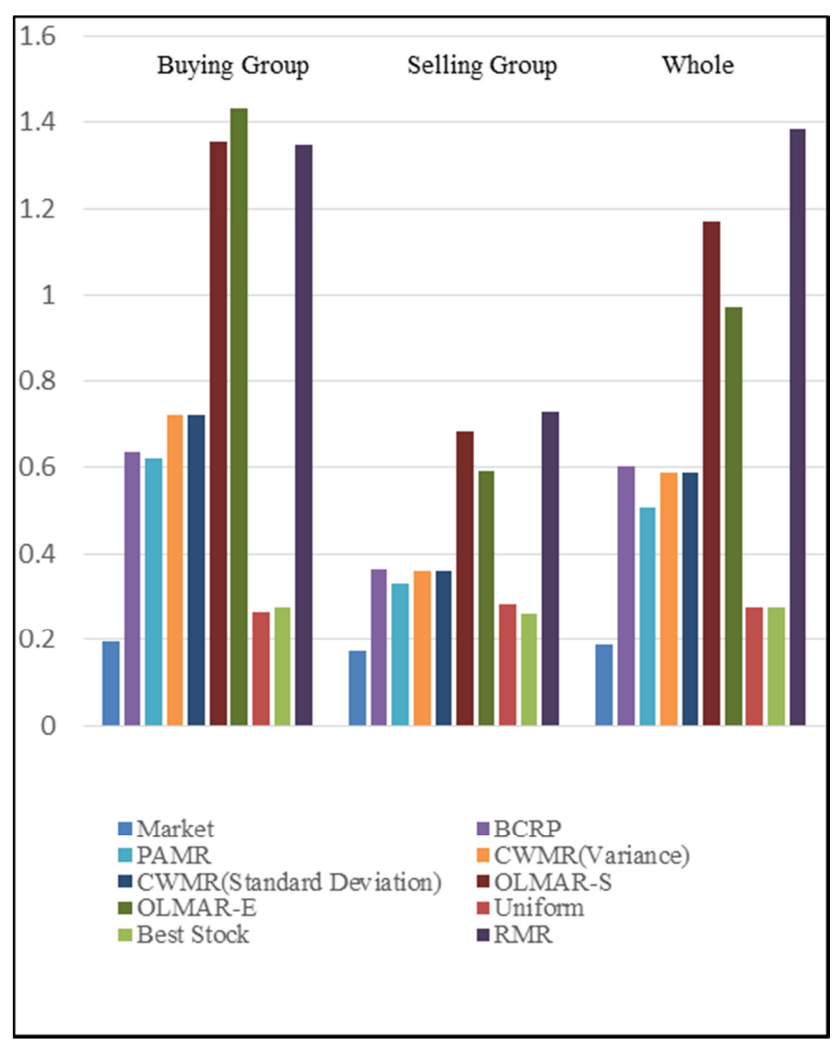

Figure 7. Calmar Ratio.

So far, we have seen that the RMR strategy for the entire group is far superior to those the two groups. We used accounting information at the beginning of the portfolio formation according to Piostroski's methodology. For the rest of the period we do not need accounting information.

In order to apply the results of this paper in practice, we should consider the transaction costs of daily portfolio adjustment and the possibility of leveraging when high returns are expected. We would like to leave the two possibility as a subject for next research.

From the observations above, we may conclude that it is worthwhile to adopt active loser following strategies. And from among active lose following strategies, we may choose either RMR or OLMAR strategies. In addition we have to consider the fact that it takes at least 5 years to let the parameters in the model to settle down and usable in later periods.

\section{Conclusion}

For a value stock portfolio, Piotroski's F-SCORE is used to construct buying stock group and selling stock group. Six follow 
the loser online portfolio strategies are applied to each group and the whole portfolio. RMR strategy for the whole portfolio has far superior performance in terms of the total cumulative return, the Sharpe ratio and Calmar ratio. It is not recommended to divide the portfolio into buying and selling groups for the value stock portfolio. Each value stock seems to secure mean reverting or trend following properties for the given preiod.

The advantage of adopting online portfolio strategy is that there is no need to use every year's accounting information as far as investment strategies are concerned. Accounting information is required only at the beginning of the portfolio formation. Also it should be noted that it takes significant amount of time till the parameters of the online portfolio strategies are stabilized. This should be true even when we do not confine the target portfolio.

From the perspective of draw down possibilities, MDD of the suggested online portfolio strategies are worse than those of the benchmark strategies, which essentially implies that machine learning does not bring us any free lunch. We have to suffer from possibility of loss of the capital whenever we want to adopt better return strategies.

\section{References}

[1] Piotroski, Joseph D. "Value investing: The use of historical financial statement information to separate winners from losers." Journal of Accounting Research (2000): 1-41.

[2] Fama, Eugene F., and Kenneth R. French. "The Cross - Section of Expected Stock Returns." the Journal of Finance 47.2 (1992): 427-465.

[3] Fama, Eugene F., and Kenneth R. French. "Size and Book to - Market Factors in Earnings and Returns." The Journal of Finance 50.1 (1995): 131-155.

[4] Rosenberg, B., Reid, K. and Lanstein, R. Persuasive Evidence of Market Inefficiency. The Journal of Portfolio Management, Institutional Investor Journals, 11, (1985), 9-16.

[5] Lakonishok, Josef, Andrei Shleifer, and Robert W. Vishny. "Contrarian investment, extrapolation, and risk." The journal of finance 49.5 (1994): 1541-1578.

[6] Piotroski, Joseph. "The impact of management forecasts on short-term stock price volatility." (2002).

[7] Ou, Jane A., and Stephen H. Penman. "Financial statement analysis and the prediction of stock returns." Journal of accounting and economics 11.4 (1989): 295-329.

[8] Holthausen, Robert W., and David F. Larcker. "The prediction of stock returns using financial statement information." Journal of Accounting and Economics 15.2-3 (1992): 373-411.

[9] Lev, Baruch, and S. Ramu Thiagarajan. "Fundamental information analysis." Journal of Accounting Research (1993): 190-215.

[10] Abarbanell, Jeffery S., and Brian J. Bushee. "Abnormal returns to a fundamental analysis strategy." Accounting Review 1998, p.19-45.

[11] Cover, Thomas M. "Elements of Information Theory Thomas M. Cover, Joy A. Thomas Copyright $(\subset) 1991$ John Wiley \&
Sons, Inc. Print ISBN 0-471-06259-6 Online ISBN 0-471-20061-1." (1991).

[12] Helmbold, David P., Darrell DE Long, and Bruce Sherrod. "A dynamic disk spin-down technique for mobile computing." Proceedings of the 2nd annual international conference on Mobile computing and networking. ACM, 1996.

[13] Helmbold, David P., et al. "On - Line Portfolio Selection Using Multiplicative Updates." Mathematical Finance 8.4 (1998): 325-347.

[14] Gaivoronski, Alexei A., and Fabio Stella. "Stochastic nonstationary optimization for finding universal portfolios." Annals of Operations Research 100.1-4 (2000): 165-188.

[15] Li, Bin, et al. "PAMR: Passive aggressive mean reversion strategy for portfolio selection." Machine learning 87.2 (2012): 221-258.

[16] Li, Bin, et al. "Confidence Weighted Mean Reversion Strategy for Online Portfolio Selection." AISTATS. 2011.

[17] Li, Bin, et al. "Confidence weighted mean reversion strategy for online portfolio selection." ACM Transactions on Knowledge Discovery from Data (TKDD) 7.1 (2013): 4.

[18] Li, Bin, and Steven CH Hoi. "Online portfolio selection with moving average reversion." (2012) 1206-4626.

[19] Huang, Dingjiang, et al. "Robust median reversion strategy for online portfolio selection." Proceedings of the Twenty Third International Joint Conference on Artificial Intelligence (2013): 2006-2012.

[20] Huang Dingiiang et al. "Combination forecasting reversion strategy for online portfolio selection" ACM Transaction on Intelligent System and Technology. 9, 5. (2018).

[21] Györfi, László, et al. A distribution-free theory of nonparametric regression. Springer Science \& Business Media, 2006.

[22] Gyorfi, Lszl, and Gyrgy Ottucsak. "Sequential prediction of unbounded stationary time series." IEEE Transactions on Information Theory 53.5 (2007): 1866-1872.

[23] Györfi, László, Frederic Udina, and Harro Walk. "Nonparametric nearest neighbor based empirical portfolio selection strategies." Statistics \& Decisions International mathematical journal for stochastic methods and models 26.2 (2008): 145-157.

[24] Liang, Zhipeng, et al. "Adversarial Deep Reinforcement Learning in Portfolio Management" arXiv:1808.09940v3 [q-fin. PM] 18: (2018)

[25] Obeidat, Samer et al. "Adaptive Portfolio Asset Allocation Optimization with Deep Learning", International Journal on Advances in Intelligent Systems, 11, 1 \& 2 (2018):25-34.

[26] Vovk, V. G. "Universal forecasting algorithms." Information and Computation 96.2 (1992): 245-277.

[27] Saunders, Craig, Alexander Gammerman, and Volodya Vovk. "Ridge regression learning algorithm in dual variables." (1998): 515-521.

[28] Das, Puja, and Arindam Banerjee. "Meta optimization and its application to portfolio selection." Proceedings of the 17th ACM SIGKDD international conference on Knowledge discovery and data mining. ACM, 2011. 
[29] Vovk, Volodya, and Chris Watkins. "Universal portfolio selection." Proceedings of the eleventh annual conference on Computational learning theory. ACM, pp.1998.

[30] Sloan, R. "Do stock prices fully reflect information in accruals and cash flows about future earnings? (Digest summary)." Accounting review 71.3 (1996): 289-315.

[31] Sweeny, A. "Debt-Covenant Violations and Managers' Accounting Responses." Journal of Accounting and Economics 17 (1994): 281-308.

[32] Myers, Stewart C., and Nicholas S. Majluf. "Corporate Financing and Investment Decisions When Firms Have Information That Investors Do Not Have." Journal of Financial Economics 13.2 (1984): 187-221.

[33] Miller, M., and K. Rock. "Dividend Policy under Asymmetric Information." Journal of Finance 40 (1985): 1031-1051.

[34] Bondt, Werner FM, and Richard Thaler. "Does the stock market overreact" The Journal of finance 40.3 (1985): 793-805.

[35] Poterba, James M., and Lawrence H. Summers. "Mean reversion in stock prices: Evidence and implications." Journal of financial economics 22.1 (1988):27-59.

[36] Lo, Andrew W., and A. Craig MacKinlay. "When are contrarian profits due to stock market overreaction." Review of Financial studies 3.2 (1990): 175-205.

[37] Borodin, Oleg, et al. "Molecular dynamics study of the influence of solid interfaces on poly (ethylene oxide) structure and dynamics." Macromolecules 36.20, 2003, p.7873-7883.

[38] Borodin, Allan, Ran El-Yaniv, and Vincent Gogan. "Can We Learn to Beat the Best Stock." J. Artif. Intell. Res. (JAIR) 21 (2004, 579-594.

[39] Duchi, John, et al. "Efficient projections onto the 11-ball for learning in high dimensions." Proceedings of the 25th international conference on Machine learning. ACM, 2008.

[40] Sharpe, William F. "Capital asset prices: A theory of market equilibrium under conditions of risk." The journal of finance 19.3 (1964): 425-442.

[41] Sharpe, William F. "The sharpe ratio." Journal of portfolio management 21.1 (1994): 49-58.

[42] Magdon-Ismail, Malik, et al. "On the maximum drawdown of a Brownian motion." Journal of applied probability 41.1 (2004): 147-161.

[43] Kahn, R., and R. Grinold. "Active Portfolio Management." New York, NY: McGraw-Hill (1999). 